\title{
Effect of Inductive Loads on IGBT Based Digital Voltage Controllers
}

\author{
Jyoti Lalotra ${ }^{1}$, Saleem Khan ${ }^{2}$, Shavet Sharma ${ }^{3}$, Parveen Lehana ${ }^{4}$ \\ ${ }^{I}$ M.Tech Student, Department of EEE, Arni University, India \\ ${ }^{2} P h D$ Student, Department of Physics and Electronics, University of Jammu, India \\ ${ }^{3}$ Assistant Professor, Department of EEE, Arni University, India \\ ${ }^{4}$ Associate Professor, Department of Physics and Electronics, University of Jammu, India
}

\begin{abstract}
Power systems with low harmonic distortion are the key requirement in the modern electronic power systems. Investigations are carried out to analyze the effect of load (combination of resistive and inductive) on the designed IGBT based power system. Harmonic distortions in the input and output voltage of the system are calculated. Input and output voltages waveforms were recorded for all combinations by using signal recording software having duration of $1 \mathrm{~s}$ with sampling rate of 16,000. Computer algorithm is designed to calculate Total Harmonic Distortion (THD) in the system. Reduction in the THD was seen in the designed microcontroller and IGBT based power system.
\end{abstract}

Keywords: THD, impedance, power system, load effect, harmonics.

\section{Introduction}

Power electronics is need of our which is developing extensively in industry, transportation, residential, commercial and in aerospace application [1-3]. The power semiconductor device was firstly introduced in 1950's [4] [5]. Nowadays, power electronics becomes important field in electrical engineering as demand of using power electronics devices such as inverter, converter and power supplies in daily life are increasing day by day. Many power electronics converter are on switching mode because the power semiconductor devices can be considered as controllable ideal switches that can turn on and off according to the corresponding gate signal.

There are some popular power devices such as Gate-turn-off-Thyristor (GTO), insulated-gate-bipolar transistor (IGBT), Metal-oxide-semiconductor field effect transistors (MOSFETs) [6-8] and MOS-controlled thyristor (MCT). Power system harmonic are not new fact. It is mainly caused by saturation of loads such as transformers, industrial arc furnaces, cables, switching mode power supplies and other devices [9-10]. Harmonic is a sinusoidal component of periodic wave or quantity having a frequency that is integer multiple of the fundamental frequency. An AC periodic voltage or current can be represented by Fourier series of pure sinusoidal waves which contain the fundamental frequency and its multiple is called harmonic [11]. A term called distortion factor or harmonic factor is often used to express the amount of harmonic distortion. It can be used to express the amount of voltage distortion or current distortion in a system. The distortion factor is determined as follows [12].

$$
D F=\left[\left(\sum X_{\text {harmonic }}{ }^{2}\right) /\left(X_{\text {Fundamental }}{ }^{2}\right)\right]^{\frac{-1}{2}} \times 100 \%
$$

The purposed research work is carried out to investigate the effect of impedance i.e. change in the inductance keeping resistance constant on IGBT based digital voltage controllers. Signal processing technique is used to evaluate the effect of the impedance and calculation of total harmonic distortion.

\section{Causes and Effect of Harmonic Distortion}

Harmonics are electric voltages and currents that appear on the electric power system as a result of non-linear electric loads. Harmonic frequencies in the power grid are a frequent cause of power quality problems. Harmonic components should be reduced as much as possible. These are causes of harmonic distortion can be described as:

\subsection{Causes of Dips, Sags and Surges}

Rural location remote from power source, unbalanced load on a three phase system, switching of heavy loads, long distance from a distribution transformer with interposed loads, unreliable grid systems and equipments not suitable for local supply.

\subsection{Causes of Transients and Spikes}

Lightening, arc welding, switching on heavy or reactive equipments such as motors, transformers, motor drives and electric grade switching, Non-linear loads, power electronic devices, load switching [13]. 


\subsection{Effects of Harmonics on Telecom Interferences}

Telephone interference is also major problems formed due to harmonics. Telephone communication is in frequency range of $20 \mathrm{~Hz}$ to $5000 \mathrm{~Hz}$. Harmonic voltage and current travels through $\mathrm{AC}$ and DC overhead transmission lines conductors. Telephone interferences produce due to electromagnetic wave of harmonics current and voltage of telephone communication frequency range $(20 \mathrm{~Hz}$ to $5000 \mathrm{~Hz})[14][15]$.

\subsection{Effect of Harmonic on Generator}

The effect of harmonic current and voltage distortion on generator is increase the heating of machine caused by increasing the iron losses, copper losses and both frequency dependent [16].

\subsection{Effect of Harmonic on Power Factor}

The existence of harmonic in power system can occur problems in power factor such as voltage distortion, increased losses and heating, and malfunction of protective equipment[17] [18].Current harmonic do not improve the power factor and reduce THD as much as their conventional two-stage counterpart.

\subsection{Effect of Harmonic on Line and Cable}

The effect of harmonic on line and cables has same problems in case of transformer, motors and generator. It increase the copper losses and heating to damage the line and cables due to appearance of skin effect this is main problems due to harmonic occur [12].

\section{Methodology}

The purposed research work can be explained in the form of block diagram as shown in Fig. 1. It comprises of six blocks: voltage measurement circuits, microcontroller based stabilizer, input voltage level shifting, variable load section, load voltage level shifting, sound card with PC and signal processing unit. Input voltage is applied to voltages measurements circuit. The operation is started by taking the $230 \mathrm{~V}, 50 \mathrm{~Hz}$ ac supply. Voltage measurements are done at the input and output section of microcontroller based stabilizer which consists of current transformer (CT). The second block is the microcontroller based stabilizer. In this block the elevation or drop in the input voltage caused by the fluctuation are stablized consisting of microcontroller and driver circuits. The microcontroller used in this circuit is dsPIC30F2010. All decisions regarding stabilization of the power are taken by the microcontroller. The microcontroller is connected to the stabilization which contains IGBT (CT60), TLP250 gate driving circuit of IGBT and other peripheral electronics components. The output from microcontroller based stabilizer is applied to load. It consists of series combinations of resistor and inductor.

Different combinations of impedance are taken as shown in Table I. Eight combinations of the load and inductances ranging from approximate value of $250 \mathrm{mH}$ to $1200 \mathrm{mH}$ are taken by keeping resistance $36 \Omega$. The input and output voltage are recorded in the PC using sound card and input voltage level shifting block. Further these signals are stored and processed in PC through sound card. The amplitude of AC voltage is very high and thus cannot be directly applied to the sound card. Circuit shown in Fig. 2 developed to bring down the voltage level from hundreds of volts to millivolts. The operation is started by taking the $230 \mathrm{~V}, 50 \mathrm{~Hz}$ ac supply. These signals are recorded in the PC using Gold Wave software and processed using digital signal processing toolbox. The sampling rate and duration of measurement are kept at value of 16000 and $1 \mathrm{~s}$ respectively.

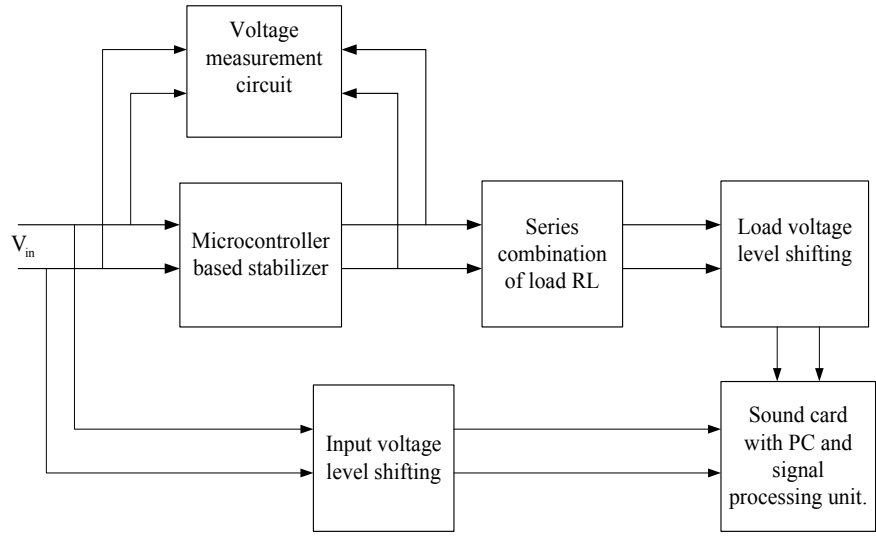

Fig. 1 Schematics block diagram of the experiment. 
Table I. Eight Different Combinations of Load RL

\begin{tabular}{|c|c|c|}
\hline S. No. & $\mathbf{R}(\boldsymbol{\Omega})$ & $\mathbf{L}(\mathbf{m H})$ \\
\hline 1 & 36 & 1232.0 \\
\hline 2 & 36 & 1073.0 \\
\hline 3 & 36 & 873.0 \\
\hline 4 & 36 & 729.5 \\
\hline 5 & 36 & 590.6 \\
\hline 6 & 36 & 470.2 \\
\hline 7 & 36 & 363.0 \\
\hline 8 & 36 & 269.1 \\
\hline
\end{tabular}

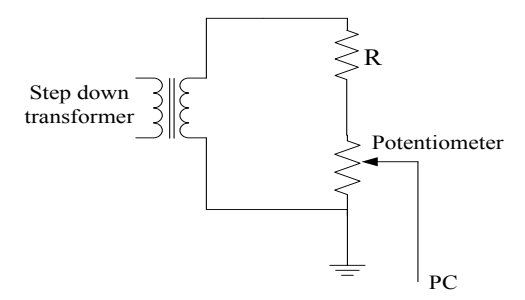

Fig.2 Voltage Step down circuit to input signal to PC.

Input and output signals THD are calculated by using the signal processing technique. The flow diagram of the designed computer algorithm to calculate the input and output value of THD is shown in Fig. 3.

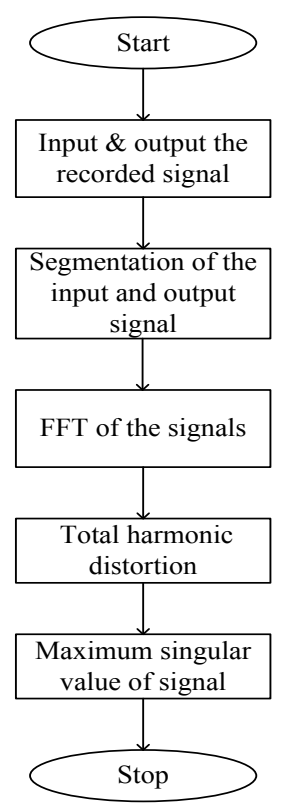

Fig. 3 Algorithm flow chart to calculate input and output signal of THD.

\section{Result and discussion}

Investigations are carried out to analyse the effect of loads (combination of resistive and inductive) on the IGBT based power system and analysis of harmonic distortion in the input and output voltages caused by different combinations of inductive and resistive load. Combinations of RL load are taken in the experiment. Input and output voltages for various combinations were recorded at 16,000 sampling rate for limited time duration of $1 \mathrm{~s}$. The signals were processed using signal processing technique to evaluate the harmonic distortion. Segment of the signals are taken and harmonic distortion in them are calculated. Fig. 4(a) to Fig. 4(h) represents the segmented input and output voltage waveforms for eight different combinations of the load with duration of $0.3 \mathrm{~s}$. Impedance of the load is calculated by using following formula given below with all the values of the inductor and resistor taken in the experiment.

$$
Z=\sqrt{R^{2}+\left(X_{L}\right)^{2}}
$$

Where $X_{L}=2 \pi f L$

The calculated value of impedance, input and output THD for eight different load values are given in Table II. Also calculated experimentally values of input and output of THD are plotted in Fig. 5. As the values 
of the impedance is decreased i.e. variation in the value of inductance from Henry $(\mathrm{H})$ to $\mathrm{mH}$, THD in the input signal shows the maximum and minimum values of 0.3027 and 0.1898 . Similarly THD, maximum and minimum value in the output voltage is 0.2196 and 0.1465 respectively. Difference in input and output value of THD is seen in the plot for certain combinations which may arise due to leakage of the inductive components. From the calculated values of THD in input and output it is observed that the harmonic distortion in the output voltage is comparatively less than the input voltage.

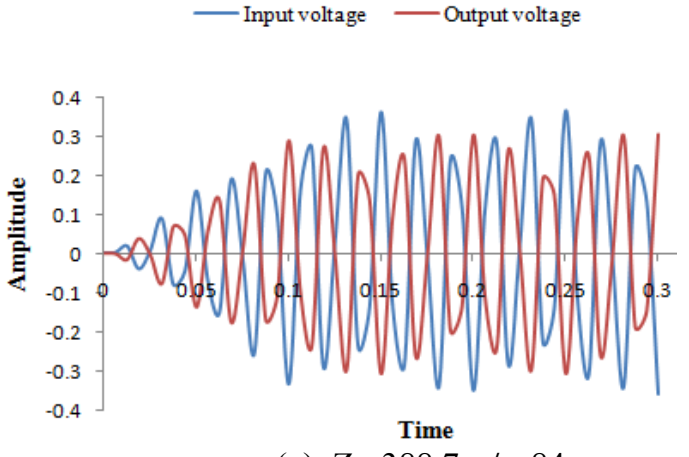

(a). $Z=388.7, \phi=84$

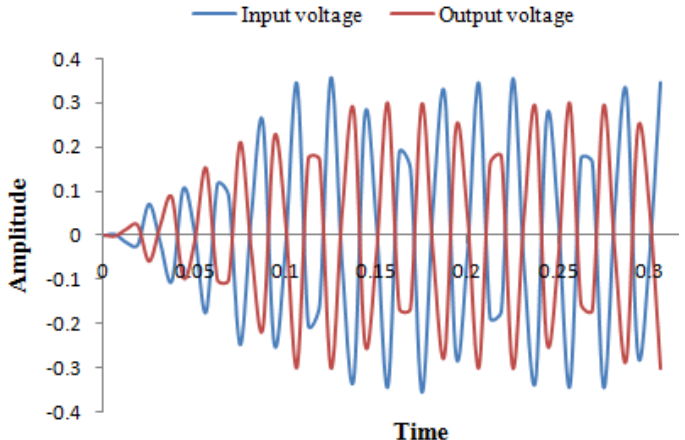

(c). $Z=276.6, \phi=82$

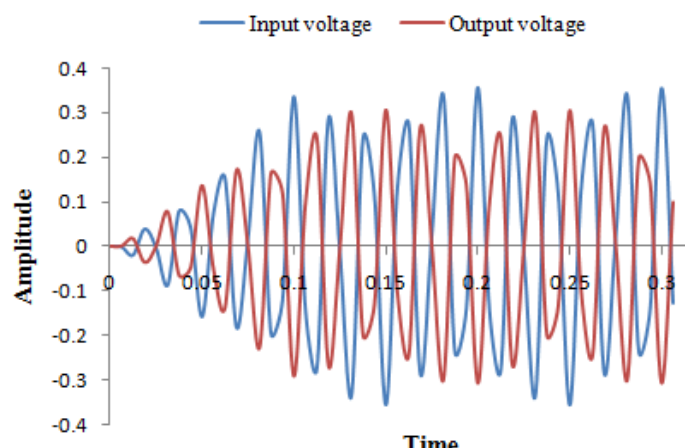

(e). $Z=189.0, \phi=79$

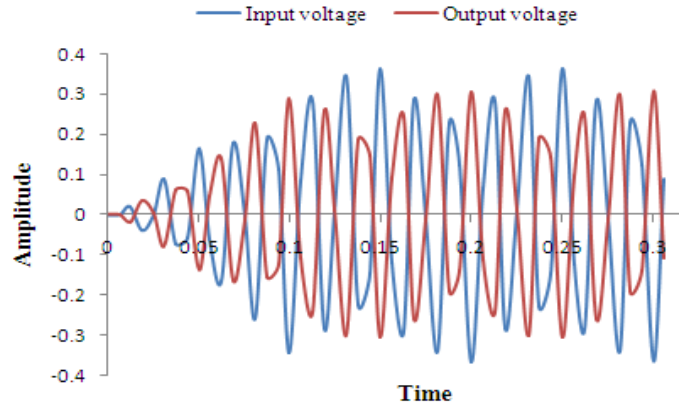

(g). $Z=119.6, \phi=72$

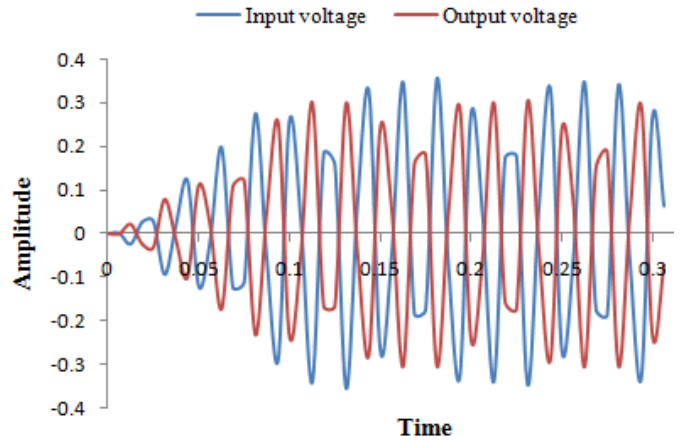

(b). $Z=339.0, \phi=83$

- Input voltage -Output voltage

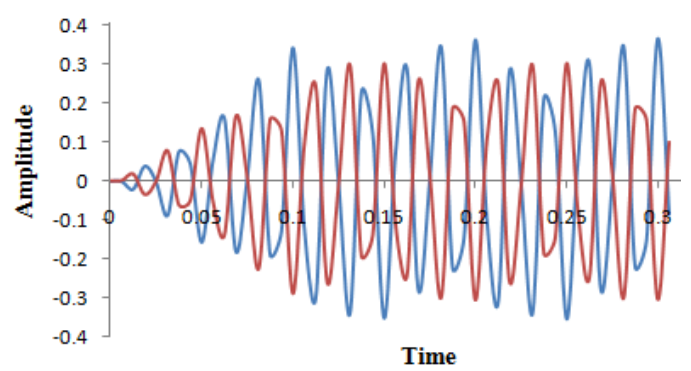

(d). $Z=232.0, \phi=81$

- Input voltage — Output voltage

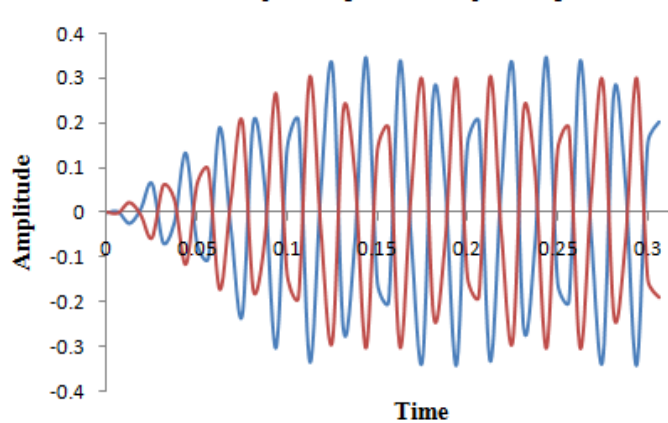

(f). $Z=152.0, \phi=76$

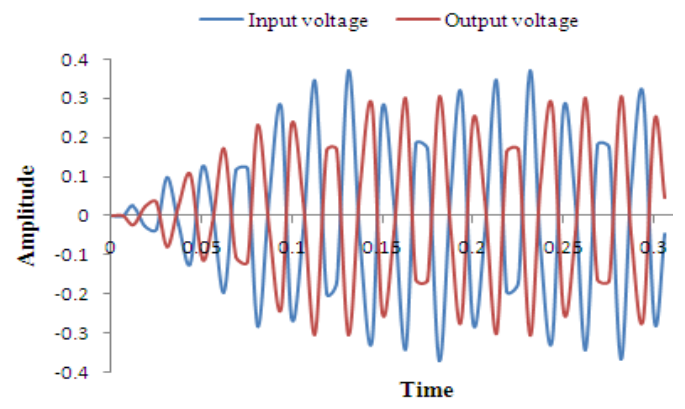

(h). $Z=91.9, \phi=66$

Fig. 4(a-h) Input and output voltage signals are recorded at 16,000 sampling rate for eight different values of inductance and constant values of resistance. 
Table II. Input and Output Value of THD

\begin{tabular}{|c|c|c|c|}
\hline S. No. & $\begin{array}{c}\text { Impedance } \\
(\mathbf{Z})\end{array}$ & Input THD & Output THD \\
\hline 1 & 388.7 & 0.2732 & 0.2063 \\
\hline 2 & 339.0 & 0.2181 & 0.1737 \\
\hline 3 & 276.6 & 0.1898 & 0.1465 \\
\hline 4 & 232.0 & 0.3027 & 0.2196 \\
\hline 5 & 189.0 & 0.2927 & 0.2175 \\
\hline 6 & 152.0 & 0.1961 & 0.1562 \\
\hline 7 & 119.6 & 0.2955 & 0.2175 \\
\hline 8 & 91.9 & 0.2681 & 0.1906 \\
\hline
\end{tabular}

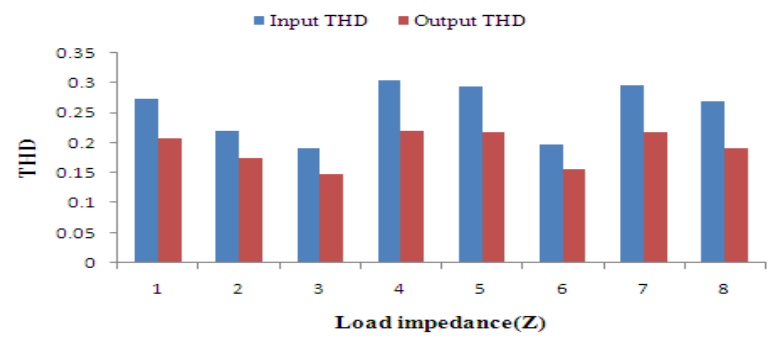

Fig.5 Experimental values of input and output of THD with different impedance values.

\section{Conclusion}

Research work is carried out to study the effect of loads (combination of resistive and inductive) on the IGBT based power system causing harmonic distortion. Eight different combinations of the load are taken with different values of inductance keeping resistance constant. Input and output voltages were recorded for all combinations using Gold Wave software with sampling rate of 16,000 and duration $1 \mathrm{~s}$. Computer algorithm is designed to evaluate THD in the input and output voltage of the designed power system with the help of digital signal processing technique. The designed microcontroller and IGBT based power system reduced the distortion in the output giving maximum value of THD in the input as 0.3027 with corresponding output THD is 0.2196 .

\section{References}

[1] N. Mohan, T.M. Undeland and W.P. Robbins, Power electronics: converters, applications and design, John Wiley \& Sons, Inc., 2003.

[2] C. Reitsma, Student Member, "An introduction to inverters and application."

[3] Toshiba Field Effect Transistor Silicon N Channel MOST Type 2SK2232, 1998, Toshiba Semiconductor, [Online], Retrieved: August 12, 2005.

[4] Daniel W. Hart, Introduction to Power Electronics, Prentice Hall, 1997.

[5] N.N. Barsoum and F.T. Png, "High quality ac power with triac inverter," CD Proceeding of Australian University in Power and Energy conference (AUPEC), Melbourne, Austraila, 2006.

[6] P. Potta, N. Srinivasan and R. Balakrishnan, "Harmonic analysis for distribution systems," International Technology Research Letters, 1(1), 2012.

[7] A. Gonzalez and J.C. MeCall, "Design of filters to reduce harmonic distortion in industrial power system," IEEE Transactions on Industry Applications, 23(3), 1987, 504-511.

[8] Francisco C. De La Rosa, Harmonics and Power Systems, Taylor \& Francis Group, CRC Press 2006, 1-184.

[9] A.E. Emanuel, "Summary of IEEE standard 1459: definitions for the measurement of electric power quantities under sinusoidal, non sinusoidal, balanced, or unbalanced conditions," IEEE Transactions on Industry Applications, 40(3), $2004,869-876$.

[10] N. Senthilnathan and T. Manigandan,“ Virtual harmonic analyser harmonic analysis and estimation of THD using lab view," Proceedings of the International Conference on Man-Machine Systems (ICOMMS), Batu Ferringhi, Penang, MALAYSIA, 2009, 4B1-1-4B1-6.

[11] M. Ahmed Mahar, Md. Aslam Uqaili and A. Sattar Larik, "Harmonic analysis of ac-dc topologies and their impacts on power systems," Mehran University Research Journal of Engineering \& Technology, 30(1), 2011, 173-178.

[12] J.P. Nelson, "A better understanding of harmonic distortion in the petrol chemical industry," IEEE Transactions on Industry Applications, 40(1), 2004, 1-14.

[13] S. Jain, S. Singh Thakur and S.P. Phulambrikar, "Improve power quality and reduce the harmonics distortion of sensitive load," International Journal of Engineering Research and Applications (IJERA), 2(6), 2012, 806-815.

[14] Francisco C. De La Rosa, Fundamentals of Harmonic Distortion and Power Quality Indices in Electric Power Systems, Harmonics and Power Systems, Copyright by Taylor \& Francis Group, LLC, 2006.

[15] A. Priyadharshini., Hans John D'cruz, B. Naresh, J. Ragul and S. Swathy, “Application of wavelet transform for detection and minimization of harmonic using shunt active filter," International Journal of Advanced Electrical and Electronics Engineering (IJAEEE), 2(3), 2013, 23-28.

[16] S. Rao, EHV-AC, HVDC Transmission \& Distribution engineering, Khanna publishers, 2011.

[17] ABS Guidance Notes on Control of Harmonics in Electrical Power Systems, American Bureau of Shipping, $2006,29-54$.

[18] J.S. Tepper, J.W. Dixon, G. Venegas and L. Moran, “A simple frequency independent method for calculating the reactive and harmonic current in a nonlinear load,” IEEE Transactions on Industry Power Electronics, 43(6), 1996, 647-653. 